\title{
Antioxidant Activity of a Oryzanols Concentrate by Differential Scanning Calorimetry
}

\author{
Bruno Irigaray $^{1}$, Iván Jachmanián ${ }^{1} \&$ María A. Grompone ${ }^{1}$ \\ ${ }^{1}$ Departamento de Ciencia y Tecnología de los Alimentos, Facultad de Química, Universidad de la República, \\ Montevideo, Gral. Flores 2124, Uruguay \\ Correspondence: Bruno Irigaray, Departamento de Ciencia y Tecnología de los Alimentos, Facultad de Química, \\ Universidad de la República, Montevideo, Gral. Flores 2124, Uruguay. E-mail: birig@fq.edu.uy
}

Received: October 31, 2017

Accepted: November 17, 2017 Online Published: December 8, 2017

doi:10.5539/jfr.v7n1p38

URL: https://doi.org/10.5539/jfr.v7n1p38

\begin{abstract}
Oryzanols are natural antioxidants that are found in appreciable amounts in rice bran oil. However, by chemically refining the crude rice bran oil they are lost during the chemical neutralization step leaving the oil refined with very little oryzanols. The chemical neutralization leaves a residue called "soapstocks" where most of these antioxidants are found. From the soapstocks and by relatively simple procedures it is possible to obtain a oryzanols concentrate which may contain $33 \%$ of them. However, its antioxidant power has been little studied in oils compared to other natural antioxidants. Therefore, the present work gives information about the antioxidant power of a concentrate of oryzanols compared to natural antioxidants such as tocopherol and synthetic antioxidants such as butylhydroxytoluene (BHT) added in oils with different degrees of unsaturation and without antioxidants. The results determined by the differential scanning calorimetry method show that the antioxidant power was variable according to the method used. The tocopherol protected the oils from the oxidation at $130^{\circ} \mathrm{C}$ (soybean and high oleic sunflower) better than the oryzanols concentrate by the isothermal method. When the non-isothermal method was used it was found that the-tocopherol protected soybean oil better than oleic high sunflower oil compared to the oryzanols concentrate. However, when comparing BHT with oryzanols concentrate, BHT generally had a lower protection in both oils and both methods. These results show that the oryzanols concentrate has a protective effect of the oxidation of the studied oils, however, this could depend on the degree of the oil unsaturation.
\end{abstract}

Keywords: oils, antioxidants, oxidation, DSC

\section{Introduction}

Different methods have been used for the determination of the oxidative stability of oils. Among them is the Differential Scanning Calorimetry (DSC) method. The basic fundamentals of the application of the DSC to the study of the different physicochemical processes that can happen in a sample and their relation with the heat flow delivered by the instrument were described by Höne, Hemminger, \& Flammersheim, 2003.

This method is based on the accelerated oxidation of the fat or oil under study. In order to study the oxidation kinetics and the oxidative stability of various fats, the isothermal and non-isothermal methods can be used (Tan, Che Man, Selamat, \& Yusoff, 2002, Rudnik, Szczucinska, Gwardiak, Szulc, \& Winiarska, 2000). This is possible because the lipid oxidation process is exothermic, so that the amount of heat released by a sample subjected to said process can be linked to the rate at which it passes (Litwinienko \& Kasprzycka-Guttman, 1998; Litwinienko, Daniluk, \& Kasprzycka-Guttman 1999; Thurgood, Ward, \& Martini, 2007). The original theoretical basis of this method was described by Ozawa (1965) and later by several authors as Shadidi and Wanasundara (1997), Simon (2006).

Therefore, the study of lipid oxidation by the isothermal method by DSC involves tracking the heat flow curve as a function of time. This allows to determine the moment at which the oxidation processes in the fatty material begin to accelerate. In this way, for the isothermal method an oxidation induction time value is defined as OIT (Simon, 2006) whereas for the non-isothermal method an oxidation temperature called OT (Moser, 2009; Dunn, 2005).

Several authors have used this methodology to monitor the protective power of antioxidants in different fatty 
materials (Litwinienko \& Kasprzycka-Guttman, 1998; Litwinienko et al., 1999; Polavka, Paligová, Cvengros, \& Simon 2005; Thurgood et al., 2007).

An important advantage of this method is the small amount of sample needed for the study, much smaller than for conventional methods (OSI and Rancimat): with quantities of about $100 \mathrm{mg}$ can be obtained the physicochemical parameters related to the oxidation kinetics, activation energy and the Arrhenius constant.

The oryzanols have an antioxidant power already studied by many researchers (Cicero \& Gaddi 2001; Wu, Beecher, Holden, Haytowitz, Gebhardt, \& Prior, 2004). Studies on the activity of oryzanols as natural antioxidants have demonstrated the effectiveness of these compounds to increase the oxidative stability of other oils when added to them in very small quantities (Warner et al., 2005). This antioxidant activity is attributed to the presence of the ferulate group in its chemical structure. It should be considered that the presence of different groups esterified in a phenolic antioxidant may play an attenuating role or antioxidant capacity enhancement (López, Laguerre, Lecomte, Figueroa, Barea, Weiss, Decker, \& Villeneuve, 2009). Therefore, it is important to study and compare its antioxidant power with that of other antioxidants in different types of oils.

In this work the antioxidant power of oryzanols concentrate was determined when they were added to oils of different origin and were compared with that of different natural and synthetic antioxidants (BHT, $\delta$-tocopherol). By means of isothermal and non-isothermal DSC oxidation some kinetic parameters of the process were determined.

\section{Materials and Methods}

\subsection{Materials}

The determinations were performed on commercially refined soybean oil and high oleic sunflower samples from Uruguayan processing. The antioxidants used (BHT and $\delta$-tocopherol) were purchased from Sigma-Aldrich. The oryzanols concentrate was prepared in the laboratory by the procedure mentioned by Irigaray, Vieitez, Jachmanián, \& Grompone (2014).

\subsection{Preparation of Antioxidant-Stripped Commercial Oils}

Commercial oils were purified on a column packed with activated $\left(200{ }^{\circ} \mathrm{C}\right.$ for $\left.3 \mathrm{~h}\right)$ aluminium oxide [28] at an oil/alumina ratio of $1: 1$. The entire system was wrapped in aluminium foil to prevent oxidation, and connected to a vacuum pump.

\subsection{Preparation of oils added with antioxidants}

The oils purified by treatment with alumina were subsequently added with three antioxidants: Butylhydroxytoluene (BHT), $\delta$-tocopherol and a 33\% purity oryzanols concentrate. For this purpose, solutions of these antioxidants were prepared in isopropanol. Subsequently, solutions of known concentration were added to the purified oils reaching an effective concentration of 3x10-3 $\mathrm{M}$ of antioxidant. The antioxidant oil solutions were placed on ultrasound for better dispersion and homogenization.

\subsection{Fatty Acid Composition Determinations}

Fatty acid composition as methyl esters was analyzed for the different oils on a Shimadzu 2010 plus gas chromatograph coupled to a Supelco SP 2330 capillary column fitted with an FID 340 flame ionization detector operating at $340{ }^{\circ} \mathrm{C}$. Injection was performed at $250{ }^{\circ} \mathrm{C}$ and $60 \mathrm{kPa}$, using $\mathrm{N}_{2}$ as carrier gas at a split ratio of 80:1.

\subsection{Oxidation Determinations by Istothermal DSC}

A TA Instruments DSC Q20 differential scanning calorimeter (DSC) was used. Using a Pasteur pipette, one drop of sample (ca. $15 \mathrm{mg}$ ) was placed in an aluminium crucible, which was then placed without lid in the DSC sample furnace. An identical, empty crucible was placed in the reference compartment. The furnace, maintained under oxidizing atmosphere with an oxygen flow of $50 \mathrm{~mL} / \mathrm{min}$, was heated at a rate of $40{ }^{\circ} \mathrm{C} / \mathrm{min}$ up to the selected temperature and kept at the final temperature for $240 \mathrm{~min}$.

\subsection{Oxidation Determinations by Non-isothermal DSC}

A similar procedure to that of isothermal DSC was used, except that all samples were placed in the DSC at a temperature of ca. $35{ }^{\circ} \mathrm{C}$, heating at different rates $\left(10,15{ }^{\circ} \mathrm{C} / \mathrm{min}\right.$ or $\left.20^{\circ} \mathrm{C} / \mathrm{min}\right)$ up to a final temperature of $250{ }^{\circ} \mathrm{C}$.

\section{Results y Discussion}

Table 1 shows the fatty acid composition of the two oils used and the corresponding inherent stability calculated 
on the basis of each composition. The oils were chosen for their important difference in inherent stability.

Table 1. Fatty acids composition of the oils used

\begin{tabular}{ccc}
\hline Fatty acid & Soybean & High oleic sunflower \\
\hline $16: 0$ & 11.2 & 3.6 \\
$18: 0$ & 3.9 & 3.1 \\
$18: 1$ & 21.0 & 87.1 \\
$18: 2$ & 55.0 & 4.8 \\
$18: 3$ & 6.6 & - \\
\hline Estabilidad inherente & 7.4 & 1.4 \\
\hline
\end{tabular}

To these oils a purification was made for the removal of the antioxidants present in them according to that mentioned in section 2.2 .
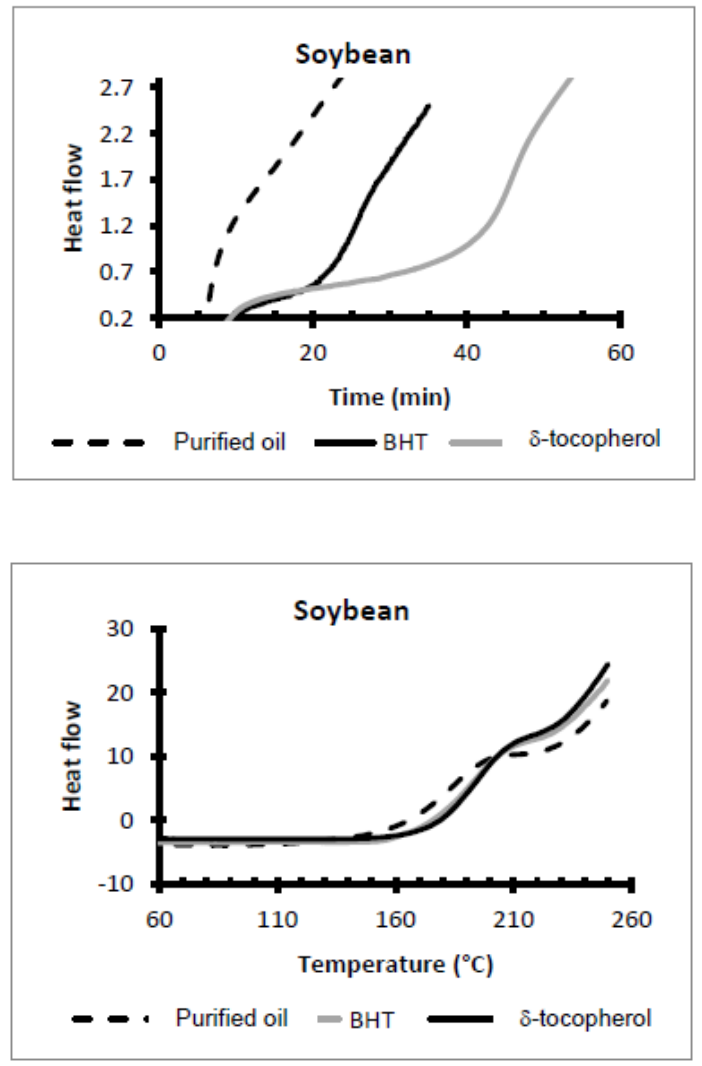

(b)

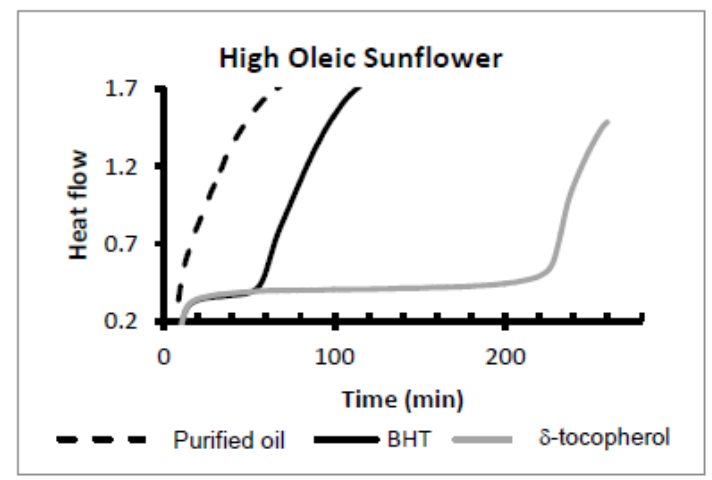

(a)

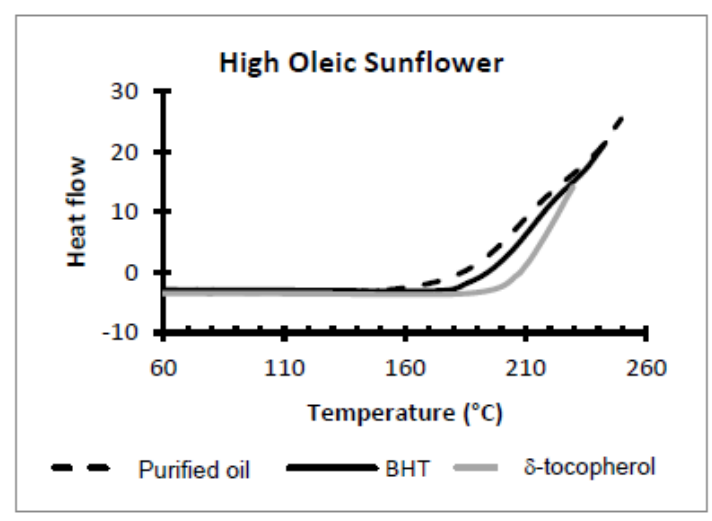

Figure 1. Heat flow curves obtained by DSC from two commercial oils purified and with antioxidant addition: a) isothermal oxidation at $130{ }^{\circ} \mathrm{C}$ and b) non-isothermal oxidation at $10 \% \mathrm{~min}$

The oils without antioxidants were oxidized by the isothermal and non-isothermal methods (figure 1). Figure 1a shows the oxidation of soybean oil and high oleic sunflower oil by the isothermal method. The thermogram for soybean oil and high oleic sunflower purified had no induction time. This could be explained by the lack of antioxidants in both oils which exert a protective effect at the beginning of the oxidation. However, in both oils with added antioxidants it was possible to determine the induction time (OIT). This arises as a consequence of a change in the oxidation rate during the course of the analysis.

When the non-isothermal method was used, an induction period was observed for both antioxidant-free and antioxidant-added oils as a change in oxidation rate between the start and end of the study (Figure 1b). In this way, the oxidation temperature (OT) could also be determined for oils without antioxidants.

With both isothermal and non-isothermal oxidations, it is possible to calculate the physicochemical parameters associated with the process, considering that both kinetics follows the Arrhenius equation (Šimon, Kolman, 
Niklová, \& Šchmidt, 2000).

For isothermal oxidation the equation that links the kinetic parameters (activation energy and pre-exponential factor of Arrhenius) is the following:

$$
\ln \frac{1}{O I T}=\ln A^{\prime}-\frac{E_{a}^{\prime} * R}{T}
$$

where $E_{a}^{\prime}$ is the apparent energy of activation $(\mathrm{kJ} / \mathrm{mol}), \mathrm{R}$ the gas constant $(\mathrm{kJ} / \mathrm{mol} . \mathrm{K})$ and $\mathrm{A}^{\prime}$ the apparent "pre-exponential" Arrhenius factor. Thus, by plotting $\ln (1 / \mathrm{OIT})$ against (1/T) a line is obtained whose slope the apparent activation energy $\mathrm{E}_{\mathrm{a}}^{\prime}$ and the apparent pre-exponential factor $\mathrm{A}^{\prime}$ can be calculated (Figures 2 and 3).

Litwinienko et al. (1998 and 1999) proposed a development that allows to determine from this analytical method the energies of activation of the oxidation process in non-isothermal DSC. This method is based on the one described by Osawa-Flynn-Wall; and has been applied in numerous research works in this area (Kasprzycka-Guttman \& Odzeniak, 1992). Combining kinetic expressions in non-isothermal systems with those corresponding to heat transfer phenomena, different researchers have contributed by a very complex development to the deduction of practical equations applicable to the determination of the kinetic parameters of a reaction occurring under these conditions (Kasprzycka-Guttman, Jaorsz-Jarszewska, \& Litwinienko, 1995; Litwinienko \& Kasprzycka-Guttman, 1998; Thurgood et al., 2007). Accordingly, the equation that links the kinetic parameters with the heating rate is as follows:

$$
\log \beta=\frac{a}{T}+b
$$

where, $\beta$ is the heating rate, $\mathrm{T}$ is the oxidation start temperature "OT" expressed in degrees Kelvin and a and $\mathrm{b}$ are parameters of the equation. Therefore, by plotting $\log \beta$ versus $(1 / \mathrm{T})$, the coefficients a (slope of the line) and $\mathrm{b}$ (ordered at the origin) can be determined, as shown in Figures 1 and 2.

Litwinienko et al. $(1998,1999)$ and Thurgood et al. (2007) related the activation energy to the slope value of the logarithm of the heating rate versus $1 / \mathrm{T}$, according to the following equation:

$$
a=-\frac{E_{a}^{\prime}}{2.19 * R}
$$

where $\mathrm{E}_{\mathrm{a}}^{\prime}$ is the apparent energy of activation $(\mathrm{kJ} / \mathrm{mol})$ and $\mathrm{R}$ is the gas constant $(\mathrm{kJ} / \mathrm{mol} . \mathrm{K})$.

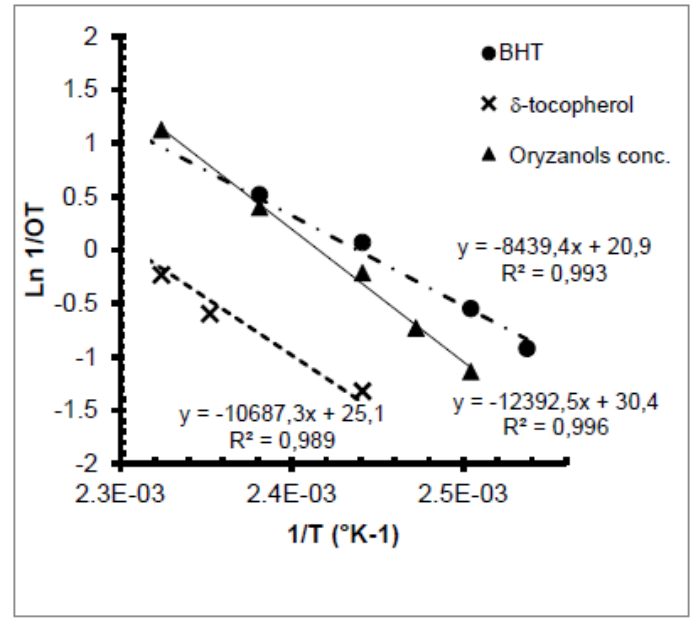

(a)

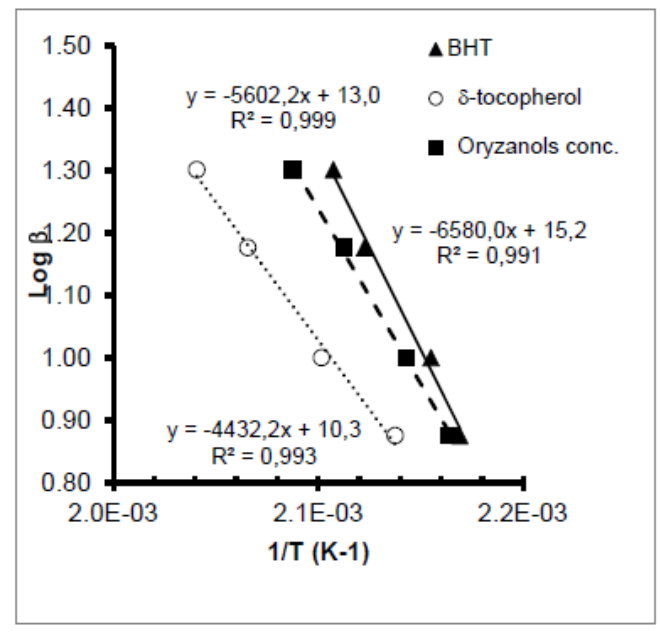

(b)

Figure 2. Correlations for high oleic sunflower oil with addition of BHT, $\delta$-tocopherol and oryzanols concentrate: a) Isothermal DSC and b) Non-isothermal DSC 


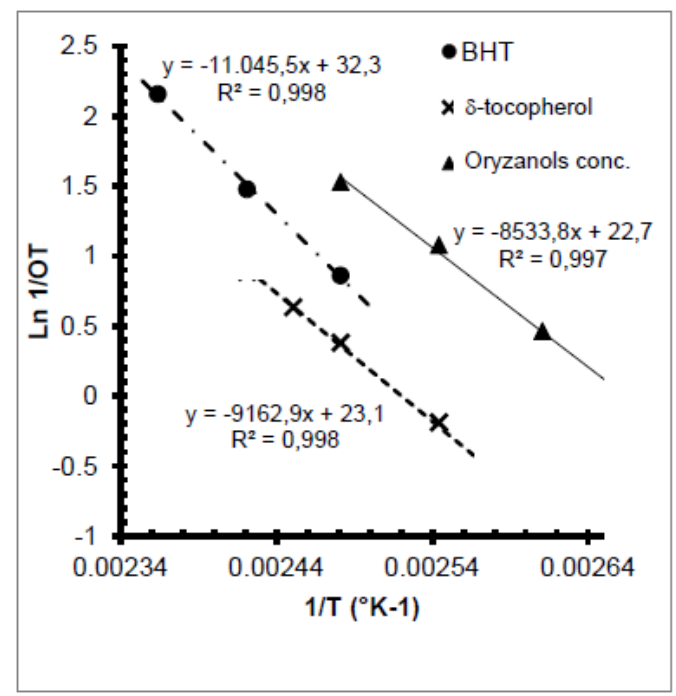

(a)

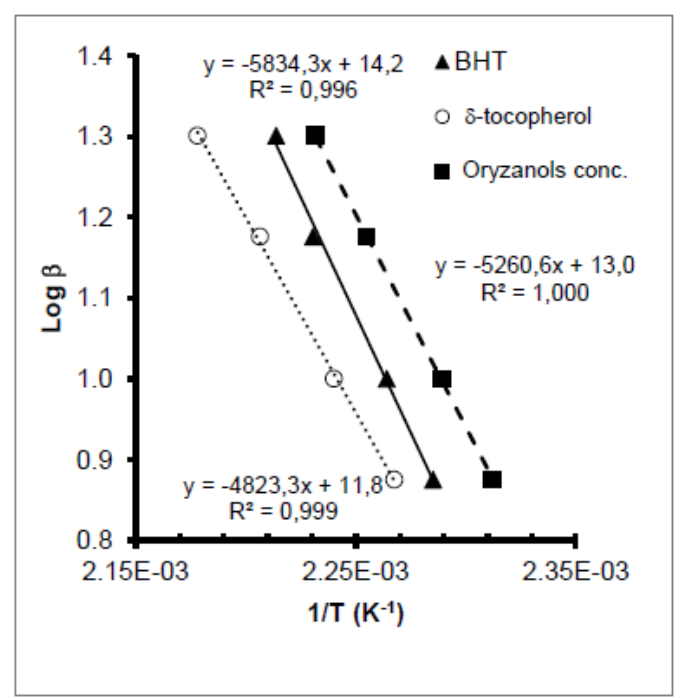

(b)

Figure 3. Correlations for soybean oil with addition of BHT, $\delta$-tocopherol and oryzanols concentrate: a) Isothermal DSC and b) Non-isothermal DSC

Litwinienko et al. (1999) and Ulkowski, Malgorzata and Litwinienko (2005) among other researchers described the relationship between the ordinate at origin "b" and the pre-exponential factor of the Arrhenius equation, by the following equation:

$$
b=-2.315+\log \frac{A^{\prime} * E^{\prime}}{R}
$$

where $E_{a}^{\prime}$ is the "apparent" activation energy $(\mathrm{kJ} / \mathrm{mol}), \mathrm{R}$ is the gas constant and $\mathrm{A}^{\prime}$ is the "apparent" pre-exponential factor of Arrhenius. Therefore, the apparent kinetic parameters can be calculated from the lines of Figures 1 and 2.

For the same oil with the addition of different antioxidants, the lines are different, that is, both the ordinate at the origin and the slope (both for the non-isothermal and the isothermal). Also, for both oils studied with the addition of the same antioxidant, the slope and the ordinate at the origin are different depending on the method used (the correlation coefficients of the lines are high). This reflects the differences in the existing oxidation kinetics since the slope is directly related to the $\mathrm{E}_{\mathrm{a}}^{\prime}$ and the ordinate at the origin with $\mathrm{A}^{\prime}$.

Table 2. Activation energy and pre-exponential factor for oils with and without added antioxidants: a) isothermal and b) non-isothermal.

\begin{tabular}{ccccc}
\hline & \multicolumn{2}{c}{ Soybean } & \multicolumn{2}{c}{ High Oleic Sunflower } \\
\cline { 2 - 5 } & $\mathrm{Ea}(\mathrm{kJ} / \mathrm{mol})$ & $\mathrm{A}\left(\mathrm{min}^{-1}\right)$ & $\mathrm{Ea}(\mathrm{kJ} / \mathrm{mol})$ & $\mathrm{A}\left(\mathrm{min}^{-1}\right)$ \\
\hline BHT & 92 & $1.7 \times 10^{12}$ & 70 & $2.0 \times 10^{7}$ \\
$\delta$-tocoferol & 76 & $1.8 \times 10^{8}$ & 89 & $1.3 \times 10^{9}$ \\
Oryzanols concéntrate & 71 & $1.2 \times 10^{8}$ & 103 & $2.7 \times 10^{11}$ \\
\hline & \multicolumn{3}{c}{ (a) } & \\
\hline & \multicolumn{3}{c}{ Soybean } & High Oleic Sunflower \\
\cline { 2 - 5 } & $\mathrm{Ea}(\mathrm{kJ} / \mathrm{mol})$ & $\mathrm{A}\left(\mathrm{min}^{-1}\right)$ & $\mathrm{Ea}(\mathrm{kJ} / \mathrm{mol})$ & $\mathrm{A}\left(\mathrm{min}^{-1}\right)$ \\
\hline Without antioxidants & 65 & $5.1 \times 10^{7}$ & 88 & $7.9 \times 10^{9}$ \\
BHT & 106 & $2.6 \times 10^{12}$ & 120 & $2.1 \times 10^{13}$ \\
$\delta$-tocopherol & 88 & $1.3 \times 10^{10}$ & 81 & $4.6 \times 10^{8}$ \\
Oryzanols concéntrate & 96 & $2.0 \times 10^{11}$ & 102 & $1.7 \times 10^{11}$ \\
\hline
\end{tabular}

(b)

Table 2 shows the activation energy and pre-exponential factor for soybean and high oleic sunflower oils with and without antioxidant aggregation by isothermal and non-isothermal methods. By the non-isothermal method, 
the activation energy of each purified oil (without antioxidants) is less or almost equal to that corresponding with the addition of antioxidants. For soybean oil, the highest activation energy corresponds to BHT (by both methods). For high oleic sunflower oil, the highest activation energy corresponds to the oryzanols concentrate (isothermal method) or to the BHT (non-isothermal method). Therefore, for this oil both methods did not present similar qualitative results.

For both oils with the addition of BHT, $\delta$-tocopherol and the oryzanols concentrate, the values of the activation energy are in the range of $70 \mathrm{~kJ} / \mathrm{mol}$ to $120 \mathrm{~kJ} / \mathrm{mol}$. When the activation energies were compared for the same oil with the addition of the same antioxidant, both methods lead to different activation energies values. The values corresponding to the pre-exponential factor also presented differences, although of several orders of magnitude. This indicates that both methods are not comparable, with respect to the determined stabilities.

Kowalski (1989) reports "apparent" activation energy values determined for the oxidation of various oils with their antioxidants by differential non-isothermal scanning calorimetry. Soybean oil with antioxidants had an "apparent" activation energy of $67.4 \mathrm{~kJ} / \mathrm{mol}$ while for common sunflower oil the value was 84.6 $\mathrm{kJ} / \mathrm{mol}$. Tan et al., 2001 using the isothermal method reported values for the "apparent" activation energy for a soybean oil with its antioxidants of $80.4 \mathrm{KJ} / \mathrm{mol}$. According to Kowalski (1991) the soybean oil had an activation energy of the order of $62 \mathrm{~kJ} / \mathrm{mol}$ by the non-isothermal method. The values found in this work are within those of the cited bibliography.

The values of the pre-exponential factor calculated by Kowalski (1989) for these oils were between $2.8 \times 10^{7}$ and $1.4 \times 10^{10} \mathrm{~min}^{-1}$ being of the order of magnitude of those determined in this study.

Table 3. Apparent oxidation rate constants $\left(\mathrm{k}^{\prime}\right)$ at $130{ }^{\circ} \mathrm{C}$ for soybean oil and high oleic sunflower oil with added antioxidants determined by the isothermal and non-isothermal method

\begin{tabular}{ccccc}
\hline & \multicolumn{4}{c}{$\mathbf{k}^{\mathbf{}}\left(\mathbf{m i n}^{-\mathbf{1}}\right)$} \\
\cline { 2 - 5 } & \multicolumn{2}{c}{ Soybean } & \multicolumn{2}{c}{ High Oleic Sunflower } \\
\cline { 2 - 5 } & Isotérmico & No isotérmico & Isotérmico & No isotérmico \\
\hline Without antioxidants & - & 0.182 & - & 0.034 \\
BHT & 2.2 & 0.045 & 0.017 & 0.011 \\
$\delta$-tocopherol & 0.023 & 0.053 & 0.003 & 0.016 \\
Oryzanols concentrate & 0.080 & 0.077 & 0.012 & 0.010 \\
\hline
\end{tabular}

The protective effect of an antioxidant at a given temperature is linked to its apparent rate constant $\mathrm{k}^{\prime}$ : the lower the oxidation reaction rate, ie the higher the protective effect. The parameters indicated in Table 2 allow to calculate through the Arrhenius equation the apparent oxidation rate constant $\left(\mathrm{k}^{\prime}\right)$ at a given temperature. Table 3 shows the values of $\mathrm{k}^{\prime}$ at $130{ }^{\circ} \mathrm{C}$ for oils with the different antioxidants added. According to the isothermal method, in both oils $\delta$-tocopherol presented a greater protective effect at that temperature compared to the other two added antioxidants. It produced a reduction in oxidation rate of about 5 times compared to BHT for high oleic sunflower oil whereas for soybean oil said reduction in speed was about 95 times. According to the non-isothermal method, for soybean oil the best antioxidant was BHT: it produced a reduction of 1.2 times with respect to $\delta$-tocopherol and 1.7 with respect to the oryzanols concentrate (it is 4 times with respect to the purified oil). For sunflower oil of high oleic, the lowest k' corresponds to the oryzanols concentrate: its protective effect is 1.1 times with respect to BHT and 1.6 with respect to $\delta$-tocopherol (it is 3.4 with respect to the purified oil). This would indicate that the three antioxidants have a very similar protective effect on both soybean oil and high oleic sunflower oil but that they act with relatively low effectiveness with respect to purified oils (without antioxidants).

\section{Conclusions}

A first conclusion, is that oryzanols concentrate studied by the non-isothermal method has a marked protective effect of oxidation at $130{ }^{\circ} \mathrm{C}$ with respect to oils without antioxidants. When comparing the behavior on the protective effect to the oxidation of the oils for the different antioxidants added it was observed that the results of apparent activation energy and apparent pre-exponential factor coincide with the data reported in the literature or are of the order of them. However, the values of the apparent kinetic rate of apparent oxidation obtained by both isothermal and non-isothermal methods for the oils with the addition of the same antioxidant at the same effective concentration showed marked differences. This may be due to the fact that both methods present different methodologies of analysis and therefore the results will not coincide. In this situation, the data obtained by both methods would be complementary in order to be able to conclude about the efficiency of a certain 
antioxidant. For the above mentioned, it is advisable to study the same system by various oxidation methods in order to obtain conclusive data regarding the protection process provided by a certain antioxidant.

\section{Acknowledgment}

To Ph.D. Grompone, who has recently died, for the contributions made in this work. I also thank Arrozur for the supply of soapstocks rice bran oil.

\section{References}

Cicero, A. F. G., \& Gaddi, A. (2001). Rice bran oil and gamma-oryzanol in the treatment of hyperlipoproteinaemias and other conditions. Phytotherapeutic Research, 15, 277-289. http://dx.doi.org/10.1002/ptr.907.

Dunn R. O. (2005). Effect of Antioxidants on the Oxidative Stability of Methyl Soyate (biodiesel). Fuel Proces. Technol, 86, 1071-1085. https://doi.org/10.1016/j.fuproc.2004.11.003

Höhne G. (2003). Differential Scanning Calorimetry, Springer-Verlag, Berlin. https://doi.org/10.1007/978-3-662-06710-9

Irigaray, B., Vieitez, I., Jachmanián, I., \& Grompone, M. A. (2014). Obtención de concentrados de orizanoles mediante la extracción con solventes aplicada sobre borras de neutralización modificadas químicamente. A\&G. 95, 300-308.

Kasprzycka-Guttman, T., \& Odzeniak D. (1992). Thermoanalytical Investigation of Edible Oils. Thermochim. Acta, 204(2), 303-310. https://doi.org/10.1007/s11746-999-0156-6.

Kasprzycka-Guttman, T., Jaorsz-Jarszewska, M., \& Litwinienko, G. (1995). Specific Heats and Kinetic Parameters of Thermo-Oxidative Decomposition of Peanut Oil. Thermochim. Acta 250, 197-205. https://doi.org/10.1016/0040-6031(94)01941-9.

Kowalski, B. (1989). Determination of Oxidative Stabililty of Edible Vegetable Oils by Pressure Differential Scanning Calorimetry. Thermochim. Acta 156, 347-358. https://doi.org/10.1002/ejlt.200300915

Kowalski, B. (1991). Thermal-Oxidative Decomposition of Edible Oils and Fats. DSC Studies. Thermochim. Acta, 184, 49-57. https://doi.org/10.1016/0040-6031(91)80134-5.

Litwinienko, G., Daniluk, A., \& Kasprzycka-Guttman, T. (1999). A Differential Scanning Calorimetry Study on the Oxidation of C12-C18 Saturated Fatty Acids and their Esters. J. Am. Oil Chem. Soc., 76(6), 655-657. https://doi.org/10.1007/s11746-999-0156-6.

Litwinienko, G., \& Kasprzycka-Guttman, T. (1998). A DSC Study on Thermoxidation Kinetics of Mustard Oil. Thermochim. Acta, 319, 185-191. https://doi.org/10.1016/S0040-6031(98)00410-9.

López, L. J., Laguerre, M. L., Lecomte, J., Figueroa, M. C., Barea, B., Weiss, J., Decker, E., \& Villeneuve, P. (2009). Kinetic and Stoichiometry of the Reaction of Chlorogenic Acid and Its Alkyl Esters against the DPPH Radical. J. Agric. Food Chem., 57, 863-870. https://doi.org/10.1021/jf803148z

Moser, B. R. (2009). Comparative Oxidative Stability of Fatty Acid Alkyl Esters by Accelerated Methods. J. Am. Oil Chem. Soc. 86, 699-706. https://doi.org/10.1007/s11746-009-1376-5.

Ozawa, T. A. (1965). New Method of Analyzing Thermogravimetric Data. Bull. Chem. Soc. Japan 38(11), 1881-1886. https://doi.org/10.1246/bcsj.38.1881.

Polavka, J., Paligová, J., Cvengroš, J., \& Šimon, P. (2005). Oxidation Stability of Methyl Esters Studied by Differential Thermal Analysis and Rancimat. J. Amer. Oil Chem. Soc., 82(7), 519-524. https://doi.org/10.1007/s11746-005-1103-4.

Rudnik, E., Szczucinska, A., Gwardiak, H., Szulc, A., \& Winiarska, A. (2000). Comparative Study of Oxidative Stability of Linseed Oil. Thermochim. Acta, 370(1-2), 135-140. https://doi.org/10.1016/S0040-6031(00)00781-4.

Shadidi, F., \& Wanasundara U. N. (1997) Measurements of Lipid Oxidation and Evaluation of Antioxidant Activity, in: Natural Antioxidants: Chemistry, Health Effects and Applications, Chapter 24, AOCS Press, Champaign, Illinois, p. 379.

Šimon, P., Kolman, Ĺ., Niklová, I., \& Šchmidt S. (2000). Analysis of the Induction Period of Oxidation of Edible Oils by Differential Scanning Calorimetry. J. Amer. Oil Chem. Soc., 77(6), 639-642.

https://doi.org/10.1007/s11746-000-0103-8. 
Šimon P. (2006). Induction Periods Theory and Applications. J. Therm. Anal. Calorimetry, 84(1), 263-270. https://doi.org/10.1007/s10973-005-7204-z.

Tan, C. P., Che Man, Y. B., Selamat, J., \& Yusoff, M. S. A. (2002). Comparative Studies of Oxidative Stability of Edible Oils by Differential Scanning Calorimetry and Oxidative Stability Index Methods. Food Chem., 76(3), 385-389. https://doi.org/10.1016/S0308-8146(01)00272-2

Tan, C. P., Che Man, Y. B., Selamat, J., \& Yusoff, M. S. A. (2001). Application of Arrhenius Kinetics to Evaluate Oxidative Stability in Vegetable Oils by Isothermal Differential Scanning Calorimetry. J. Am. Oil Chem. Soc., 78(11), 1133-1138. https://doi.org/10.1007/s11746-001-0401-1

Thurgood, J., Ward, R., \& Martini, S. (2007). Oxidation Kinetics of Soybean Oil/Anhydrous Milk Fat Blends: A differential Scanning Calorimetry Study. Food Res. Int. 40, 1030-1037.

https://doi.org/10.1016/j.foodres.2007.05.004.

Warner, K., \& Laszlo, J. A. (2005). Addition of Ferulic Acid, Ethyl Ferulate, and Feruloylated Monoacyl- and Diacylglycerols to Salad Oils and Frying Oils. J. Am. Oil Chem. Soc., 82, 647-652. https://doi.org/10.1007/s11746-005-1123-0.

Wu, X., Beecher, G. R., Holden, J. M., Haytowitz, D. B., Gebhardt, S. E., \& Prior, R.L. (2004). Lipophilic and hydrophilic antioxidant capacities of common foods in the United States. J. Agric. Food Chem. 52, 4016-4037. http://pubs.acs.org/doi/abs/10.1021/jf049696w.

Ulkowski, M., Malgorzata, M., \& Litwinienko G. (2005). Use of Differential Scanning Calorimetry to Study Lipid Oxidation. 1. Oxidative Stability of Lecithin and Linolenic Acid. J. Agric. Food Chem. 53, 9073-9077. http://pubs.acs.org/doi/abs/10.1021/jf051289c.

\section{Copyrights}

Copyright for this article is retained by the author(s), with first publication rights granted to the journal.

This is an open-access article distributed under the terms and conditions of the Creative Commons Attribution license (http://creativecommons.org/licenses/by/4.0/). 